\title{
Inhomogeneous imperfect fluid spherical models without Big-Bang singularity
}

\author{
Naresh Dadhich, * \\ Inter-University Centre for Astronomy \& Astrophysics, \\ Post Bag 4, Ganeshkhind, Pune - 411 007, India.
}

\begin{abstract}
So far all known singularity-free cosmological models are cylindrically symmetric. Here we present a new family of spherically symmetric non-singular models filled with imperfect fluid and radial heat flow, and satisfying the weak and strong energy conditions. For large $t$ anisotropy in pressure and heat flux tend to vanish leading to a perfect fluid. There is a free function of time in the model, which can be suitably chosen for non-singular behaviour and there exist multiplicity of such choices.
\end{abstract}

PACS numbers : 04.20Jb, 98.80Dr.

IUCAA-40/97

${ }^{*}$ E-mail : nkd@iucaa.ernet.in 


\section{Introduction}

Although the present day observations indicate that the Universe at large scale is homogeneous and isotropic and it is well described by the standard Friedman-Robertson-Walker (FRW) model. It is also a well recognised view that consideration of inhomogeneity at early times is well in order for generic initial conditions and for facilitating formation of large scale structures in the Universe. Further it is now known for some time [1-4] that inhomogeneous spacetime admits a family of perfect fluid cosmological models without the big- bang singularity. Note that these models are exact solutions of the Einstein equation satisfying the causality and energy conditions, yet there is no divergence of any physical and geometric parameters throughout the spacetime. This happens because spacetime does not admit compact trapped surfaces which invalidates application of the singularity theorems [5].

From the Raychaudhuri equation [6], it is clear that singularity can be avoided only if acceleration or rotation is non-zero. In cosmology rotation is generally not favoured and hence acceleration must be present if singularity is to be avoided. This means spacetime has to be inhomogeneous. In all the known non-singular solutions, shear is also non-zero, indicating anisotropy as well. It can be proved for a general orthogonal metric separable in space and time variables [4-5] and for a G2- symmetric perfect fluid model that presence of shear is essential for presence of acceleration [7]. Though shear contributes positively to the effective gravitational charge density in the Raychaudhuri equation, its dynamical role as making collapse incoherent can combine well with acceleration in avoiding singularity. Thus shear also seems to be playing very important role in non-singular models.

So far all known non-singular solutions are inhomogeneous and isotropic as well as have cylindrical symmetry [1-4,8-9]. It has perhaps their cylindrical symmetry that comes in the way of their application in practical cosmology. Since the Universe is known to be spherical to a good degree, it is pertinent to ask whether it is possible to have a spherically symmetric cosmological model? In this note we give a singularity-free prescription for imperfect fluid with heat flux. This is a prescription rather than a solution for no equation has been solved. A general spherically symmetric metric has only four independent stresses, which could be interpreted as density, radial and transverse 
pressures, and radial heat flux. This will be true for any spherically symmetric metric. The question is to give a prescription which is free of singularity and has proper desired behaviour for physical parameters. This has been achieved by letting the Tikekar static model [10], which is a particular case of the Tolman solution [11], expand.

Our model represents a spherically symmetric cosmological universe filled with imperfect fluid having unequal radial and transverse pressure and radial heat flow. The spacetime satisfies the weak and strong energy conditions. It is free of any kind of singularity as all the physical as well as kinematic parameters remain finite and regular in the entire range of the variables $t$ and $r$. It has the typical behaviour of a non-singular model; density and pressure vanishing for large $t$ and $r$ and being maximum at $t=0$ and $r=0$, expansion parameter and radial heat flux change their sense at $t=0$, while acceleration tends to zero as $r \longrightarrow 0$. There is however an unusual feature that heat flows radially inward as the universe expands. The heat flow vanishes at $r$ or $t \longrightarrow 0$ as well as $r$ or $\pm t \longrightarrow \infty$ and it falls off as $t^{-4}$ or $r^{-4}$ asymptotically. The pressure anisotropy vanishes for $r \longrightarrow 0$ as well as for $r$ or $\pm t \longrightarrow \infty$. That is, asymptotically it tends to perfect fluid. The shear is also non-zero and hence the model is both inhomogeneous and anisotropic.

\section{The model}

The model is described by the spherically symmetric metric,

$$
d s^{2}=\left(r^{2}+P\right) d t^{2}-\frac{2 r^{2}+P}{r^{2}+P} d r^{2}-r^{2}\left(d \theta^{2}+\sin ^{2} \theta d \varphi^{2}\right)
$$

where $P=P(t)$. The imperfect fluid is represented by the energy-momentum tensor [12],

$$
\left.T_{i k}=(\rho+p) u_{i} u_{k}-p g_{i k}+\triangle p\left[c_{i} c_{k}+\frac{1}{3}\left(g_{i k}-u_{i} u_{k}\right)\right]+2 q c_{(i} u_{k}\right)
$$

where $u_{i}$ and $c_{i}$ are respectively unit timelike and spacelike vectors, $\rho$ the energy density, $p$ the isotropic fluid pressure, $\Delta p$ the pressure anisotropy and 
$q$ heat flux.

We employ the comoving coordinates to write $u_{i}=\sqrt{g_{00}} \delta_{i}^{0}$ and take $c_{i}=$ $\sqrt{g_{11}} \delta_{i}^{1}$. The kinematic parameters; expansion, shear and acceleration for the metric (1) read as follows:

$$
\theta=\frac{-\dot{P} r^{2}}{2\left(2 r^{2}+P\right)\left(r^{2}+P\right)^{3 / 2}}, \sigma^{2}=\frac{2}{3} \theta^{2}, \quad \dot{u}_{r}=-\frac{r}{r^{2}+P} .
$$

Now applying the Einstein equation, we obtain

$$
\begin{gathered}
8 \pi \rho=\frac{2 r^{2}+3 P}{\left(2 r^{2}+P\right)^{2}} \\
8 \pi p_{r}=\frac{1}{2 r^{2}+P} \\
8 \pi p_{\perp}=\frac{1}{2 r^{2}+P}+\frac{r^{2}}{4\left(2 r^{2}+P\right)\left(r^{2}+P\right)^{2}}\left[2 \ddot{P}-\frac{\left(9 r^{2}+5 P\right) \dot{P}^{2}}{\left(2 r^{2}+P\right)\left(r^{2}+P\right)}\right] \\
8 \pi q=\frac{-\dot{P} r}{\left(2 r^{2}+P\right)^{3 / 2}\left(r^{2}+P\right)} .
\end{gathered}
$$

The pressure anisotropy $\triangle p=p_{r}-p_{\perp}$ is given by

$$
8 \pi \triangle p=\frac{-r^{2}}{4\left(2 r^{2}+P\right)\left(r^{2}+P\right)^{2}}\left[2 \ddot{P}-\frac{\left(9 r^{2}+5 P\right) \dot{P}^{2}}{\left(2 r^{2}+P\right)\left(r^{2}+P\right)}\right] .
$$

Now we just need to make a suitable choice for $P(t)$ such that all physical and kinematic parameters remain finite and regular. Any even function of $t$ without zero would be an appropriate choice, for instance $P=\alpha^{2}+\beta^{2} t^{2}$ or $\cosh ^{a} k t$ and so on. Thus there exists a family of non-singular models. In general $P(t)$ is free similar to the scale factor in FRW models. In here there is a great deal of freedom and one may as well ask that any spherically symmetric metric could be viewed as representing an imperfect fluid with radial heat flux. This is however true. Even then it is a non-trivial matter to have acceptable behaviour for model satisfying energy conditions and being 
free of singularity. As far as we know this is for the first time such a proposal has been made.

Let us consider the simplest case, $P=\alpha^{2}+\beta^{2} t^{2}$. It is easy to verify that all the above physical parameters as well as the kinematic parameters remain finite and regular for the entire range of variables; $-\infty<t<\infty, 0 \leq r<\infty$. This indicates clearly that the model is free of singularity. All the above parameters tend to zero for $t \longrightarrow \pm \infty$ and/or $r \longrightarrow \infty$. Asymptotically it is low density universe, which when contracts, attains the maximum density at $t=0$ and $r=0$, specified by the parameter $\alpha\left(\rho_{\max }=3 / 8 \pi \alpha^{2}\right)$, which can be chosen as small as one pleases to have as large $\rho_{\max }$ as desired. For large $t, \beta$ will correspond to the Hubble parameter. At $t=0$, the expansion parameter changes sign (contraction $\longleftrightarrow$ expansion) and the acceleration tends to zero as $r \longrightarrow 0$. The universe starts from low density, contracts to high density and again expands to low density state without encountering singular behaviour of any kind. Anisotropy in pressure $\triangle p$ tends to zero for $r \longrightarrow 0$ as well as asymptotically $(t \longrightarrow \pm \infty$ or $r \longrightarrow \infty)$. For small $r$ and large $t$, it approximates to the radiation universe, $\rho=3 p$, while for large $r$ it tends to an isothermal stiff fluid with $\rho=p \sim 1 / r^{2}$ [13]. $\rho$ and $p_{r}$ fall off as $t^{-2}$, whereas $\triangle p$ falls off as $t^{-6}$ and $q$ as $t^{-4}$, which indicate that fluid turns almost perfect (with isotropic pressure) for large enough $t$.

We shall now verify that the model satisfies the weak and strong energy conditions; $T_{i k} \xi^{i} \xi^{k} \geq 0$ and $\left(T_{i k}-\frac{1}{2} T g_{i k}\right) \xi^{i} \xi^{k} \geq 0$ for any non-spacelike vector $\xi^{i}$. We have

$$
T_{i k} \xi^{i} \xi^{k}=n^{2}\left(\rho+p_{r}-\triangle p\right)-m^{2}\left(p_{r}-\triangle p\right)
$$

and

$$
\left(T_{i k}-\frac{1}{2} T g_{i k}\right) \xi^{i} \xi^{k}=\frac{1}{2}\left[\left(2 n^{2}-m^{2}\right)\left(\rho+p_{r}-\triangle p\right)+m^{2}\left(2 p_{r}-\triangle p\right)\right]
$$

where $\xi_{i} u^{i}=n, \xi_{i} \xi^{i}=m^{2}$ and $n^{2} \geq m^{2}$ always. It is clear from eqns (4), (5) and (8) that the weak and strong energy conditions will be satisfied for $P(t)=\alpha^{2}+\beta^{2} t^{2}$. A lengthy but straight forward calculation shows that the model cannot however satisfy the dominant energy condition (the vector 
$T_{i k} \xi^{k}$ is non-spacelike).

All this is very fine but there is a discomforting feature indicated by $q \theta<0$ (from (3) and (7)) in general independent of specific choice for $P(t)$. This implies that there is a radially inward heat flux for expanding phase and outward for contracting phase. It may be noted that for the particular model under consideration, $q=0$ for $r$ or $t=0$ and it falls off as $r^{-4}$ or $t^{-4}$ for large $r$ or $t$. However, we must confess that this is rather an unusual feature.

The overall evolution of the model is typical of non- singular models $[3,4]$; asymptotically low density passing through the dense state at $\mathrm{t}=0$, where interchange occurs between expansion and contraction on one hand and between inflow and outflow for the radial heat flux on the other. The remarkable features of this model are: (a) it is free of big-bang as well as any other singularity, (b) it is spherically symmetric, which augurs well with the symmetry of the realistic Universe, (c) for small $r$ and large $t$ it approximates to the radiation Universe, $\rho=3 p,(\mathrm{~d})$ asymptotically $(t \longrightarrow \pm \infty$ and/or $r \longrightarrow \infty)$, the pressure anisotropy and heat flux vanish leading to perfect fluid with $\rho=3 p \sim 1 / t^{2}$ for large $t$ and $\rho=p \sim 1 / r^{2}$ for large $r$, (e) the parameter $\alpha$ defines the maximum density $\left(\rho_{\max }=3 / 8 \pi \alpha^{2}\right.$ for $\left.t=0, r=0\right)$ and $\beta$ will correspond to the Hubble parameter for large $t$, (f) the expansion parameter $\theta$ and heat flux $q$ change sign at $t=0$ and fall off to zero as $t \longrightarrow \pm \infty$ and/or $r \longrightarrow \infty$ and $q \theta<0,(\mathrm{~g})$ the acceleration $\dot{u}_{r}$ tends to zero as $r \longrightarrow 0$ and also falls off to zero asymptotically $(t \longrightarrow \pm \infty$ and/or $r \longrightarrow \infty)$, and (h) it is evidently causally stable and obeys the weak and strong energy conditions. It however does not satisfy the dominant energy condition, which may perhaps be responsible for unusual behaviour for heat flow (radially inward as the universe expands). It has also been argued that at high densities, the possibility of violation of the dominant energy condition cannot be ruled out [14]. That means at high density $d p / d \rho$ may not always measure the true signal propogation velocity and hence even if $d p / d \rho \geq 1$ there is no conflict with special relativity. Thus possibility of $p \geq \rho$ cannot be ruled out. In the context of our model, it suggests that at high density some unusual behaviour may be permissible. However the model is free of singularity and satisfies the weak and strong energy conditions.

One of the main objections against the so far known non-singular cosmo- 
logical solutions was that they did not accord to spherical symmetry and hence their application to realistic cosmology was greatly marred. By finding a family of spherically symmetric non- singular models we have overcome this objection quite successfully and hence paving way for their practical application in cosmology. It should be noted that the model is as general as FRW with $P(t)$ being free. Non-singular character will however constrain the choice for $P(t)$, but even then there would remain good deal of freedom. It would therefore be pertinent to examine its cosmological viability. This is what we would like to do next. Finally it is quite probable that there could similarly exist other families of non-singular spherical models which would make it all very exciting.

Acknowledgement : It is a pleasure to thank Roy Maartens and Sunil Maharaj for helpful discussion through e-mail. 


\section{References}

[1] J.M.M. Senovilla (1990) Phys. Rev. Lett. 64, 2219.

[2] E. Ruiz and J.M.M. Senovilla (1992) Phys. Rev. D 45, 1995.

[3] N. Dadhich, L.K. Patel and R. Tikekar (1995) Pramana : J. Phys. 44, 303.

[4] N. Dadhich, In Inhomogeneous cosmological models, eds. A. Molina and J.M.M. Senovilla (World Scientific, 1995).

[5] S.W. Hawking and G.F.R. Ellis, The Large Scale Structure of the Universe (Cambridge, 1973).

[6] A.K. Raychaudhuri (1955) Phys. Rev. 90, 1123.

[7] N. Dadhich and L.K. Patel (1997) GRG 29, 179.

[8] J.M.M. Senovilla and C.F. Sopuerta (1994) Class. Quant. Gravity. 11, 2073.

[9] M. Mars (1995) Class. Quant. Grav. 12, 2831.

[10] R.S. Tikekar (1970) Current Science 20, 460.

[11] R.C. Tolman (1939) Phys. Rev. 55, 364.

[12] S.D. Maharaj and R. Maartens (1986) J. Math. Phys. 27, 2517.

[13] W.C. Saslaw, S.D. Maharaj and N. Dadhich (1996) Ap.J. 471, 571.

[14] G. Caporaso and K. Brecher (1979) Phys. Rev. D20, 1823. 